\title{
The Detectability of Seyfert 2 Galaxies with Hidden Broad-Line Regions
}

\author{
W. $\operatorname{Bian}^{1,2}$ \\ ${ }^{1}$ Department of Physics and Institute of Theoretical Physics, Nanjing Normal University, \\ Nanjing 210097, China \\ ${ }^{2}$ Key Laboratory for Particle Astrophysics, Institute of High Energy Physics, Chinese \\ Academy of Sciences, Beijing 100039, China
}

The detectability of Seyfert 2 galaxies (Sy2s) with hidden broad-line regions(HBLRs) is still a question open to debate. Using a large sample of 90 Seyfert 2 galaxies (Sy2s) with spectropolarimetric observations ( $\mathrm{Gu} \&$ Huang 2002), we tested the suggestion that the presence of hidden broad-line regions (HBLRs) in Sy2s is dependent upon the Eddington ratio (Nicastro et al. 2003). The stellar velocity dispersion and the extinction-corrected [O III] luminosity are used to derive the masses of central super-massive black holes and the Eddington ratios. The main conclusion are summarized as follows.

- There is no obvious discrimination in Eddington ratios for Sy2s with and without HBLRs.

- The detectability of HBLRs is low for Sy2s with low-luminosity and low Eddington ratios regardless of the column density of neutral hydrogen

- For high-luminosity Compton-thin Sy2s, we find a very higher detectability of HBLRs $(\sim 85 \%)$.

In the future, we need more new data of hard X-ray spectra with good quality optical spectropolarimetric information to confirm this suggestion. Refer to Bian \& Gu (2006) for detail.

This work has been supported by the NSFC (Nos. 10403005, 10473005, 10325313, 10233030 and 10521001) and the Science-Technology Key Foundation from Education Department of China (No. 206053).

\section{References}

Bian W. \& Gu Q. 2006, ApJ, submitted

Gu Q. \& Huang J. 2002, ApJ 579, 205

Nicastro F., Martocchia A., Matt G. 2003, ApJ 589, L13

\begin{tabular}{lllllllll}
\hline $\begin{array}{l}\text { Type } \\
(1)\end{array}$ & $\log \left(M_{B H} / \mathrm{M}_{\odot}\right)$ & $\begin{array}{l}S D_{1} \\
(3)\end{array}$ & $\begin{array}{l}\mathrm{N} 1 \\
(4)\end{array}$ & $\begin{array}{l}\log \left(L_{b o l} / L_{E d d}\right) \\
(5)\end{array}$ & $\begin{array}{l}S D_{2} \\
(6)\end{array}$ & $\begin{array}{l}\mathrm{N} 2 \\
(7)\end{array}$ & $\begin{array}{l}f_{60} / f_{25} \\
(8)\end{array}$ & $\begin{array}{l}S D_{3} \mathrm{~N} 3 \\
(9)\end{array}$ \\
\hline non-HBLRs Sy2s & $7.22 \pm 0.10$ & 0.57 & 36 & $-0.47 \pm 0.16$ & 0.93 & 34 & $5.08 \pm 0.39$ & 2.6746 \\
HBLRs Sy2s & $7.33 \pm 0.10$ & 0.51 & 24 & $-0.07 \pm 0.16$ & 0.79 & 24 & $2.50 \pm 0.21$ & 1.3039 \\
A:non-HBLRs Sy2s & $7.23 \pm 0.10$ & 0.55 & 33 & $-0.46 \pm 0.17$ & 0.95 & 31 & $4.86 \pm 0.40$ & 2.6142 \\
A:HBLRs Sy2s & $7.33 \pm 0.11$ & 0.52 & 22 & $-0.04 \pm 0.16$ & 0.79 & 23 & $2.43 \pm 0.21$ & 1.2636 \\
B:non-HBLRs Sy2s & $7.24 \pm 0.13$ & 0.54 & 16 & $-0.47 \pm 0.22$ & 0.87 & 16 & $5.62 \pm 0.67$ & 2.8518 \\
B:HBLRs Sy2s & $7.34 \pm 0.13$ & 0.56 & 19 & $-0.07 \pm 0.17$ & 0.73 & 19 & $2.46 \pm 0.23$ & 1.1024 \\
C:non-HBLRs Sy2s & $7.33 \pm 0.11$ & 0.53 & 25 & $-0.78 \pm 0.19$ & 0.91 & 23 & $5.43 \pm 0.48$ & 2.8435 \\
C:HBLRs Sy2s & $7.33 \pm 0.12$ & 0.52 & 20 & $-0.17 \pm 0.16$ & 0.72 & 20 & $2.58 \pm 0.23$ & 1.3735 \\
\hline
\end{tabular}

Table 1. The distributions of SMBHs masses, the Eddington ratios and $f_{60} / f_{25}$ for HBLRs Sy2s and non-HBLRs Sy2s. A: unabsorbed Sy2s $\left(N_{H}<10^{22} \mathrm{~cm}^{-2}\right)$ excluded from the sample. B: Sy2s with $N_{H}>10^{22} \mathrm{~cm}^{-2}$. C: Sy2s with the direct $\sigma_{*}$ measurements. 